\title{
A Method For Ice Thickness Characterization Using GNSS C/N0 data
}

\author{
Ankit Regmi ${ }^{1}$, Markus Berg ${ }^{1}$, Aarno Pärssinen ${ }^{1}$ \\ Centre for Wireless Communications ${ }^{1}$, University of Oulu, Oulu, Finland, ankit.regmi@oulu.fi
}

\begin{abstract}
A Dual Circular Polarized (CP) reception method is proposed to simultaneously record direct and reflected signals in GNSS reflectometry. The purpose of using a dual $\mathrm{CP}$ antenna system is to exploit incident wave's polarization variation after reflection. This paper presents the theoretical background for dual $\mathrm{CP}$ reception system and the advantage of using dual $\mathrm{CP}$ system over a single polarization. Theoretical multipath propagation is validated by measurements performed at frozen Baltic sea. The Right-Hand Circular Polarization (RHCP) and Left-Hand Circular Polarization (LHCP) received signals are post-processed to retrieve information about the measurement environment. The analysis method gives the evidence of penetration of GNSS signal into the layered media and the possibility to characterize icethickness or layered media using only $\mathrm{C} / \mathrm{N} 0$ data.
\end{abstract}

Index Terms-GNSS-R, multipath, carrier-to-noise density ratio, dual CP antenna, reflection coefficient.

\section{INTRODUCTION AND BACKGROUND}

Global Navigation Satellite Systems (GNSS) are designed for global positioning and navigational applications. GNSS signals can be received anywhere in the world with a compatible antenna and a receiver. GNSS reception systems are mainly designed to receive direct signal and mitigate the multipath signal to provide reliable positioning solution. Multipath signals arise, when the propagated signals undergo phenomenon such as reflection, diffraction or scattering after encountering any obstacle before arriving at the receiver. The multipath signal arrives with a different path and delay with respect to direct or Line-of-Sight (LOS) signal. In a multipath rich environment, the receiver locks on to the composite signal (i.e. the combination of direct and multipath), possibly resulting in large errors in positioning. Therefore, the positioning accuracy depends on the propagation environment. The detection and mitigation of the multipath have been of great importance for the reliability of GNSS and improving positioning accuracy. Multipath was an unwanted quantity in early days for GNSS. However, for past couple of decades, the study of multipath signal has been of great interest and importance, as it opens a new paradigm of research and opportunity for GNSS Reflectometry (GNSS-R). The multipath signal carries information about the object it comes into contact with [1]. The aim is to analyze this signal to extract the characteristics of multipath environment.

GNSS satellites transmit RHCP signal. The RHCP signal undergoes approx. $180^{\circ}$ phase shift after reflection from a dielectric material and the resultant reflected signal becomes Left Hand Elliptically Polarized (LHEP). The phase reversal takes place only after the Brewster angle of the reflected medium, considering grazing incidence (satellite elevation angle). At low elevation angles (below Brewster angle), the reflected wave is RHCP i.e. no phase reversal takes place. The direct and reflected signals are coherently added resulting in a pattern of constructive and destructive interference. The study of this interference pattern have been used to retrieve several parameters for environment sensing. Several experiment-based research articles have been published in order to estimate the altimetry, soil moisture content, vegetation water content, snow depth, and wind velocity over the ocean, using GNSS-R [2][5]. These methods use a single RHCP antenna to analyze the interference behavior of the multipath signal to extract the parameter of interest. However, this technique is only valid for low elevation angles. A dual Circularly Polarized (CP) antenna technique is a new subject in GNSS-R and several research activities are going on around the world to use this system for further analysis of GNSS multipath signals and to improve positioning accuracy and environment sensing [1], [6], [7]. The key motivation of using a dual CP antenna system is the simultaneous reception of direct RHCP and reflected LHCP signals. The reflected signal can be analyzed separately enabling better understanding of multipath environment. The GNSS-R technique can be further explored using dual CP system and can be extended for all elevation angles.

In this paper, the measurements results using dual $\mathrm{CP}$ antenna system are analyzed to characterize sea-ice thickness. The measurement and data analysis considerations for dual $\mathrm{CP}$ antenna system have been explored. Analysis for both polarization have been performed. This paper is arranged in following order. Section II gives the theoretical background for the analysis of GNSS signals for both polarization. Section III describes the dual CP antenna system and its measurement considerations. The measurement scenarios are elaborated in Section IV. Analysis of measurement data and results are explained in Section V. Future improvements and agendas and conclusion are presented in Section VI.

\section{Propagation and Multipath Fading of GNSS SIGNALS}

GNSS signals are electromagnetic (EM) waves transmitted at various system-dependent frequency bands. In this study, only Global Positioning System (GPS) L1 frequency band with centre frequency $1.575 \mathrm{GHz}$ is considered. Behavior of EM waves depends on the characteristics of the medium (dielectric properties), geometry of the propagation environment, angle of incidence, and the frequency of the signal. Upon interaction with certain media, GNSS signals undergo phenomenon such 
as reflection, diffraction and scattering. This study focuses only on reflection of GNSS signals. The reflected signal with respect to the direct LOS signal will travel excess distance and reach the receiver with different path and delay. The amplitude and phase of reflected wave depends on the electromagnetic properties of the reflecting medium and excess path length travelled by the reflected signal. In case of specular reflection, coherent addition of direct and reflected signal at the receiver gives a pattern of constructive and destructive interference. A clear interference pattern is achieved if the direct and the reflected waves have same polarization. Hence, for GNSS systems a clear interference pattern can be obtained for RHCP at low elevation angles or below the Brewster angle (considering single reflecting surface). The multipath fading expression of the received signal strength $P$ for $n$ reflections can be written as [8]

$$
P=A_{o}\left(1+\sum_{i=1}^{n} \rho_{i} e^{\left(\delta R+\psi_{i}\right)}\right),
$$

where, $A_{o}$ is the received signal strength, $\rho_{i}$ and $\psi_{i}$ are the amplitude and phase of the Fresnel reflection coefficient of the reflecting medium. The $\delta R$ is the phase change due to excess path length, which is given as [9]

$$
\delta R=\frac{4 \pi h \sin \theta}{\lambda}
$$

where $h$ is the height of the receiving antenna's phase centre measured perpendicular from the reflecting surface, $\lambda$ is the wavelength of GPS L1 signal, i.e. $0.1905 \mathrm{~m}$, and $\theta$ is the satellite elevation angle.

According to (2), the received signal's phase is the function of height of receiving antenna above the reflecting surface, the satellite elevation angle and the phase of the reflection coefficient. The continuously varying excess path length relative to the satellite orbital motion, introduces an interference pattern with certain frequency, known as multipath modulation frequency [10] and is given by

$$
f_{m u l}=\frac{2 h \cos \theta}{\lambda} \frac{d \theta}{d t} .
$$

Eq. 3 can be used to estimate the frequency of the multipath signal if the height of the antenna is known or viceversa. Snow depth and ice thickness have been extracted in previous experiments using this approach [2]. This requires the knowledge of the antenna height from the reflecting surface or multipath frequency before the snow or ice accumulates on the surface. The ice or snow thickness can easily be extracted comparing the change in multipath frequency at different stages of accumulation. However, it is a very lengthy process and may require long measurement campaigns.

A novel approach is described for the characterization of the ice-thickness using only GPS C/N0 data and dual CP antenna reception system. The propagation model is based on Multiple Ray Single Reflection (MRSR) model [11]. The total reflected signal is the sum of the specular component and the product of refracted and reflected components from consecutive boundaries (or layers), respectively. Fig.1 shows the propagation phenomenon for a three layered propagation model considered in this study.

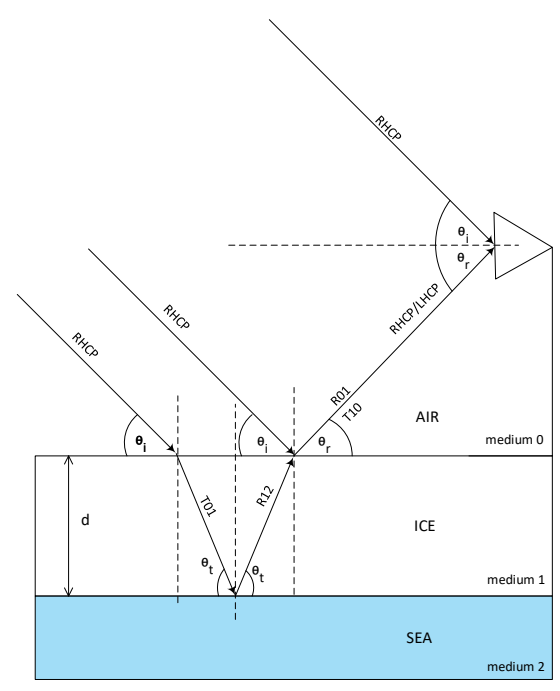

Fig. 1. MRSR propagation scenario

The first layer is air, second layer is a homogeneous layer of sea-ice with arbitrary thickness and the bottom most layer is sea water. The total reflected signal will depend on total reflection and transmission coefficients from and through all the propagating medium. Total reflection coefficient is a function of the satellite elevation angle, dielectric properties of all the propagating media (air, sea-ice and sea water), and the wavelength of the carrier wave. Coherent addition of the specular component and the reflected component from sea into ice will take place at ice-air boundary. This will result in the oscillating nature of total reflection coefficient [12]. The expression for total reflection coefficient is given by

$$
R_{t o t}=R_{01}+T_{01} R_{12} T_{10} \exp (-j 2 \delta),
$$

where $R_{i j}$ refers to the Fresnel reflection coefficient for the wave travelling in $i^{\text {th }}$ layer and incident on $j^{\text {th }}$ layer boundary, and $T_{i j}$ is the transmission coefficient for wave travelling from $i^{\text {th }}$ layer to $j^{t h}$ layer. The electrical length, $\delta$, travelled by EM wave inside the medium is given by [13]

$$
\delta=\frac{2 \pi d}{\lambda} \sqrt{e_{r}-\cos ^{2} \theta}
$$

where $d$ is the thickness of the layered medium, $\lambda$ is the freespace wavelength and $e_{r}$ is the relative permittivity of the layered medium. The exponential term in (4) accounts for the decay or attenuation of GNSS wave inside the layered media. 


\section{DUAL CP RECEPTION SYSTEM}

The dual CP antenna is used for the measurements of GNSS direct and reflected signals. The RHCP and LHCP have identical radiation pattern and can simultaneously receive direct and reflected signals at equal gains for horizontal orientation. The radiation pattern for both, RHCP and $\mathrm{LHCP}$ at phi $=0^{\circ}$ and phi $=90^{\circ}$ are shown in Fig. 2 and Fig.3. The cross-polarization discrimination (XPD) of the antenna is greater than $25 \mathrm{~dB}$. The antenna azimuth is symmetric for all elevations. The details of antenna can be found in [14].

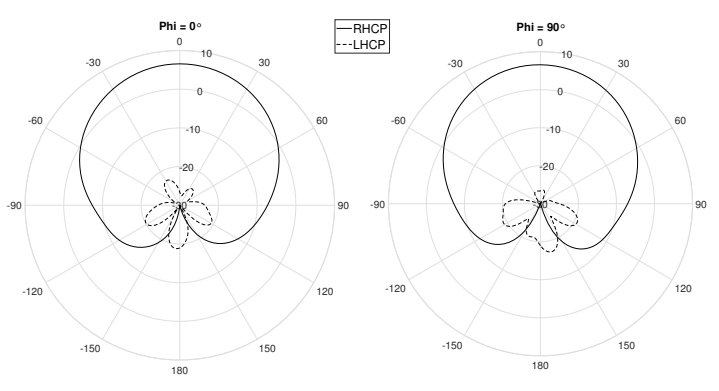

Fig. 2. RHCP Co-pol vs Cross-pol radiation pattern

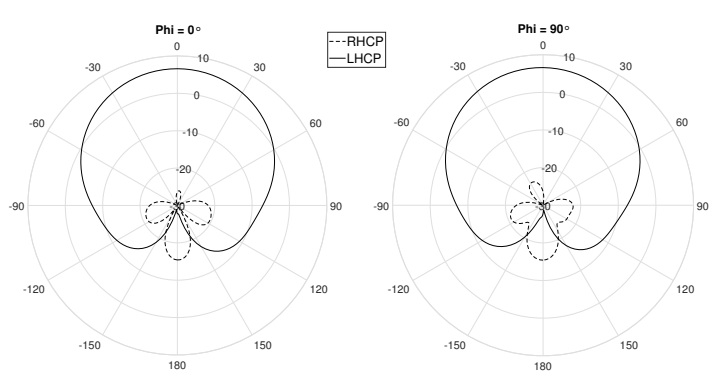

Fig. 3. LHCP Co-pol vs Cross-pol radiation pattern

The analysis of the received signal has to take into account the radiation pattern of the antenna for both polarization. The reception of both polarization are modelled as

$$
\begin{aligned}
R X_{R H C P}= & F\left(\theta_{R}\right)\left(\text { Direct }_{R H C P}+\text { Reflected }_{R H C P}\right) \\
& +F\left(\theta_{R L}\right)\left(\text { Reflected }_{L H C P}\right),
\end{aligned}
$$

and

$$
\begin{aligned}
R X_{L H C P}= & F\left(\theta_{L R}\right)\left(\text { Direct }_{R H C P}+\text { Re }_{\text {flected }} \text { fHCP }_{\text {r }}\right) \\
& +F\left(\theta_{L}\right)\left(\text { Reflected }_{L H C P}\right),
\end{aligned}
$$

where $R X_{R H C P}$ and $R X_{L H C P}$ denote signals received by RHCP and LHCP antennas, respectively. $F\left(\theta_{R}\right)$ and $F\left(\theta_{L}\right)$ denote principle gain patterns for $\mathrm{RH}$ and $\mathrm{LH}$ at angles equal to satellite elevation angles, whereas $F\left(\theta_{R L}\right)$ and $F\left(\theta_{L R}\right)$ are their respective cross polarized gain. From the above equations one can easily interpret the behavior of received single for a simple horizontal reflecting surface and known electromagnetic characteristics. According to the radiation patterns and (6) and (7), it is clear that only very small amount of crossed polarized component of signal is captured by the both ports of the antenna and its effect can vary with the environment.

\section{Measurement Setup and Scenario}

GNSS reflection measurement was performed using dual $\mathrm{CP}$ antennas and two low cost GNSS receivers. The receivers were connected to RHCP and LHCP ports of the antenna to record both signals, simultaneously. A laptop computer with receiver software was used to record data and visualize sky plot of available satellites. The used parameters given by the receiver output were satellite elevation and azimuth angles, user location and signal strength as carrier-to-noise density ratio $(\mathrm{C} / \mathrm{N} 0)$ in $\mathrm{dB}-\mathrm{Hz}$ at the rate of 1 sample per second (sample rate $1 \mathrm{~Hz}$ ). The satellites with suitable azimuth and elevation were chosen to achieve well aligned geometry with the antenna boresight. The minimum resolution of the receiver for $\mathrm{C} / \mathrm{N} 0$ data and elevation and azimuth angles were $1 \mathrm{~dB}-\mathrm{Hz}$ and $1^{\circ}$, respectively.

Measurements were performed on $12^{\text {th }}$ of February, 2019 at frozen Baltic sea, in Oulu, Finland. The measurement coordinates were $65^{\circ} 1^{\prime} 49^{\prime \prime} \mathrm{N}$ and $25^{\circ} 24^{\prime} 41^{\prime}$ " E. This region of Baltic sea is known as the Gulf of Bothnia. The salinity ranges from 2-4 PSU in these areas, which is significantly low as compared to other open sea conditions. The sea was completely frozen and ice-thickness according to Finnish Meteorological Institute data was in the range of $35-60 \mathrm{~cm}$. The measurement lasted for about 2 hours and the average temperature was $-11^{\circ} \mathrm{C}$. The measurement setup is shown in Fig.4.

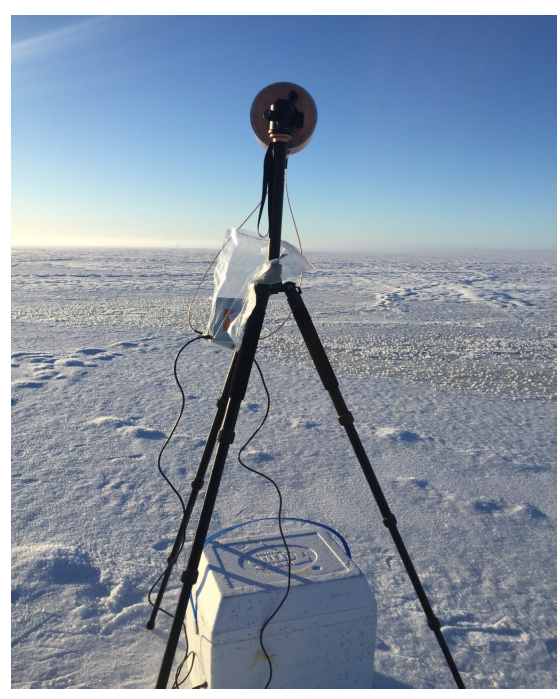

Fig. 4. Measurement setup in Oulu, Finland

The effect of a thin layer of snow present above the compact ice is not significant for this study and therefore, is not considered in the analysis.

\section{Results And AnAlysis}

Direct and reflected GNSS signals were recorded using dual CP antenna. GPS satellite PRN24 was selected for analysis 
based on most suitable geometry requirements for GNSS-R measurements. The effect of direct LOS signal is removed from the raw data using the radiation patterns shown in Fig. 2 and Fig. 3. C/NO data is converted to linear scale from $\mathrm{dB}-\mathrm{Hz}$ for better resolution and computation. The detrended voltage ratio for measured data and simulated results are shown in Fig. 5. The elevation angle below $5^{\circ}$ is not considered.

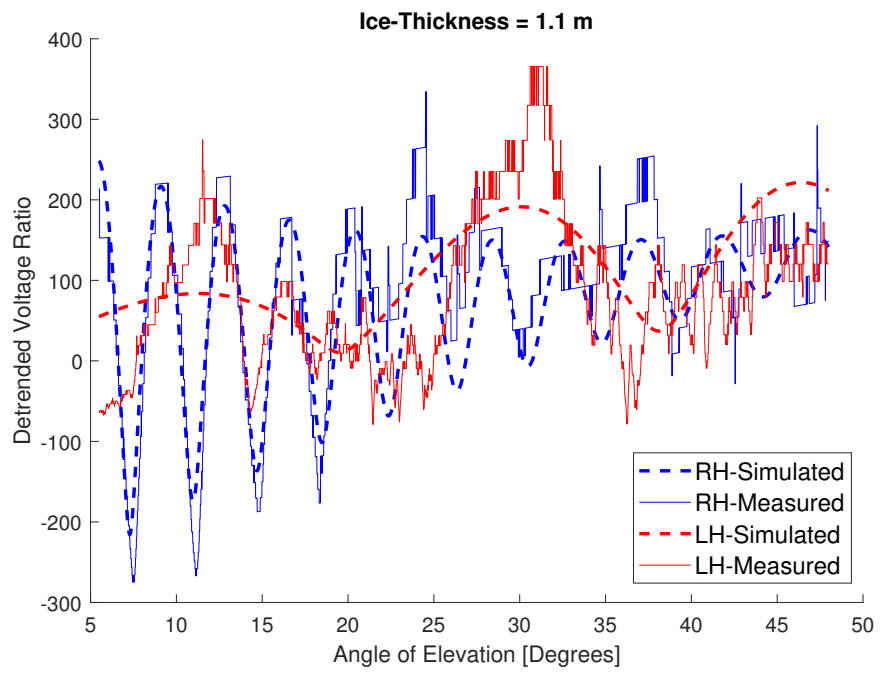

Fig. 5. Received signal strength vs Angle of Elevation

A clear interference pattern is seen in Fig. 5 for both polarization. However, the period or frequency of oscillation are completely different. The coherent addition of direct and reflected signal results in the RHCP interference pattern and its frequency is the function of antenna height above reflecting surface, satellite elevation angle and total reflection coefficient as in Eq. (1) and (4).

The direct LOS component in LH received signal is insignificant. Therefore, the LH received signal is directly the function of reflection coefficient of the reflecting surface. In case of infinitely thick homogeneous surface or highly reflective medium, the $\mathrm{LH}$ reflected signal would contain only the dominant specular reflected component. In case of sea-ice, the evidence of reflection from within the ice-layer is clearly seen in LH signal analysis. The LH received signal is the coherent addition of specular and reflected component within the ice layer and sea surface at the ice-air boundary as seen in Fig. 1 and Eq. (4) [12]. The ice-thickness of $1.1 \mathrm{~m}$ was the best fit corresponding to the measured response and was calculated using Eq. 4 and Eq. 5.

Reflection coefficient has been calculated using Fresnel Equations [8] and Eq. (4). The permittivity values are derived from [15] and are shown in Table I.

The multipath frequency of the fading signal can be calculated manually from the data or more accurately using spectral analysis. Multipath frequency can be directly related to antenna height above the reflecting surface as in Eq. (3) for RHCP. The Lomb-Scargle Periodogram (LSP) is used for frequency analysis of received signals and to extract corre-
TABLE I

MATERIAL PROPERTIES

\begin{tabular}{|c|c|}
\hline Materials & Complex Relative Permittivity \\
\hline Air & $1-j 0.0$ \\
Sea-Ice & $3.2349-j 0.0152$ \\
Sea-Water & $86.3413-j 22.3795$ \\
\hline
\end{tabular}

sponding antenna height. The LSP of the RHCP signal and extracted antenna height is shown in Fig. 6. GNSS altimetry was successfully performed with accuracy of $\pm 5 \mathrm{~cm}$, using interference pattern of RHCP signal. Here, the dominating peak gives the height of antenna above the surface and can be calculated using Eq. (3). The two secondary peaks witnessed in the measured response can be due to uneven distribution of snow or varying ice-thickness or other propagation effects that are not considered here and are beyond the scope of this study.

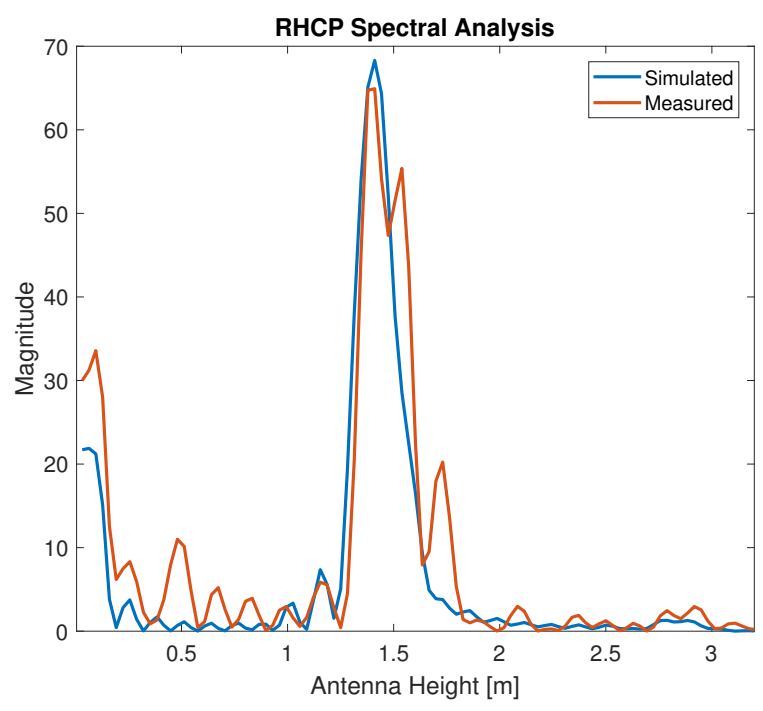

Fig. 6. Antenna height above reflecting surface

The measured and theoretical frequency responses of the LHCP antenna are shown in Fig.7. The frequency of oscillation due to sea ice layer agrees well according to measured and theoretical results. Thus, the oscillation frequency of reflection coefficient can be directly related to the thickness of the medium and relative permittivities of layered and bottom most surface. Here, ice layer is considered as a homogeneous but in reality the ice state at the surface and at the bottom (above sea) might have different water content and therefore, may have different permittivities. With the known state of ice, multiple layer model can be implemented resulting in better accuracy. The spectral analysis of multiple layers can give separate frequency components for separate layers depending on path difference with respect to the specular signal enabling us to identifying multiple layers and their thickness [11]. 


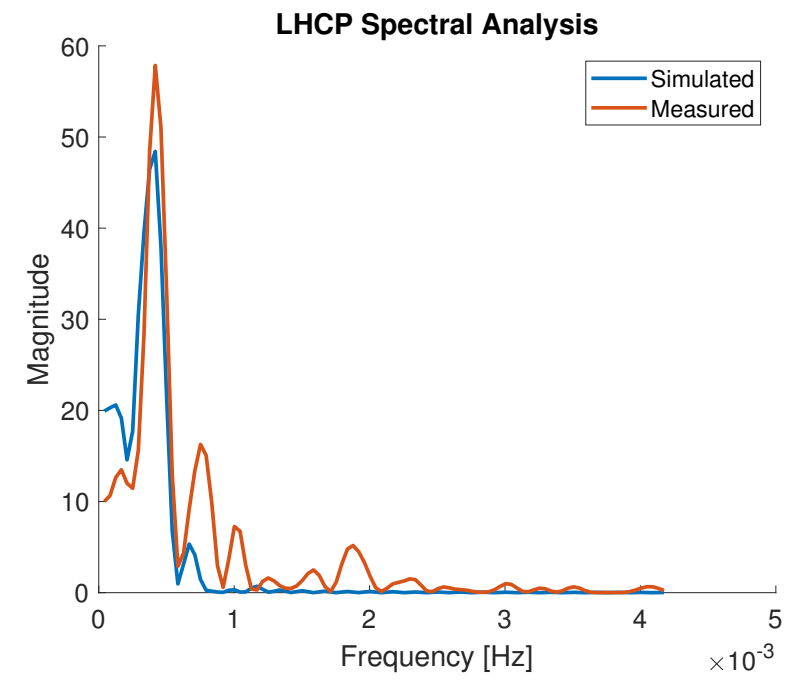

Fig. 7. LSP of LHCP signal

\section{CONCLUSION}

GNSS-R measurement was performed using dual CP antenna reception system. The polarization change of incident GNSS wave after reflection was exploited to enhance the understanding of reflecting surface. The reflected LHCP signal provided the evidence of propagation of GNSS signals through the layered sea ice. The MRSR model was implemented for theoretical analysis and it agrees very well with measured result giving the measure of ice-thickness. Thus, a simple lowcost solution for ice-thickness detection is proposed using only C/N0 data and dual CP reception system. The ice-thickness value was taken to be the best fit with the received response. Therefore, further measurements and more detailed analysis of this phenomenon is required for accurate measurement of ice-thickness or detection of layered media using GNSS-R.

\section{ACKNOWLEDGMENT}

This work was supported in part by the Academy of Finland 6Genesis Flagship (grant no. 318927).

\section{REFERENCES}

[1] D. Manandhar, R. Shibasaki, and H. Torimoto, GPS reflected signal analysis using software receiver, J. Global Positioning Syst., vol. 15, pp. 2934, 2006.

[2] Larson, K. M., E. D. Gutmann, V. U. Zavorotny, J. J. Braun, M. W. Williams, and F. G. Nievinski (2009), Can we measure snow depth with GPS receivers?, Geophys. Res. Lett., 36, L17502.

[3] Jin, Shuanggen, and Attila Komjathy. "GNSS reflectometry and remote sensing: New objectives and results." Advances in Space Research 46.2 (2010): 111-117

[4] K.M. Larson, GPS interferometric reflectometry: applications to surface soil moisture, snow depth, and vegetation water content in the western United States Wiley Interdiscip. Rev., 3 (2016), pp. 775- 787, 10.1002/wat2.1167

[5] Rodriguez-Alvarez, Nereida, et al. "Soil moisture retrieval using GNSS R techniques: Experimental results over a bare soil field." IEEE Transactions on Geoscience and Remote Sensing 47.11 (2009): 3616- 3624.

[6] M. Berg, R.U.R. Lighari, Jani Kallankari, Ville Majava, Aarno Prssinen, and Erkki T. Salonen, Polarization based measurement system for analysis of gnss multipath signals, in press, in Antennas and Propagation Conference, 10th European, Davos, Switzerland, April 2016.
[7] Jia, Yan, et al "Estimation of surface characteristics using GNSS LH reflected signals: Land versus water." IEEE Journal of Selected Topics in Applied Earth Observations and Remote Sensing 9.10 (2016): 4752 4758.

[8] A. Alonso Arroyo et al., "Dual-Polarization GNSS-R Interference Pattern Technique for Soil Moisture Mapping," in IEEE Journal of Selected Topics in Applied Earth Observations and Remote Sensing, vol. 7, no. 5, pp. 1533-1544, May 2014. doi: 10.1109/JSTARS.2014.2320792

[9] Hannah, Bruce M. (2001) Modelling and simulation of GPS multipath propagation. PhD thesis, Queensland University of Technology.

[10] Y. Georgiadou and A. Kleusberg, On carrier signal multipath effects in relative GPS positioning, Manuscripta Geodaetica, vol. 13, no. 3, pp. $172179,1988$.

[11] Estel Cardellach, Fran Fabra, Antonio Rius, Simone Pettinato, Salvatore D'Addio, Characterization of dry-snow sub-structure using GNSS reflected signals, Remote Sensing of Environment, Volume 124, 2012, Pages 122-134.

[12] Ulaby, Fawwaz T., Richard K. Moore, and Adrian K. Fung. Microwave Remote Sensing: Active and Passive. Vol. 1, Microwave Remote Sensing Fundamentals and Radiometry. Norwood, MA.: Artech House, 1981.

[13] ITU-R. 2015. Effects of building materials and structures on radiowave propagation above about $100 \mathrm{MHz}$. International Telecommunications Union. ITU-R Recommendation P.2040-1.

[14] M. Berg, J.Chen and A. Pärssinen. Radiation Characteristics of Differentially-Fed Dual Circularly Polarized GNSS Antenna. In Proc. 13th European Conference on Antennas and Propagation (EuCAP 2019), Krakow, Poland, 2019, in press.

[15] ITU-R. 1992. Electrical characteristics of the surface of the earth. International Telecommunications Union. ITU-R Recommendation P.527-3. 